Revue d'histoire de l'Amérique française

REVUE D.HISTOIRE DE L'AMÉRIQUE FRANÇAISE

\title{
Lavigne, Marie et Michèle Stanton-Jean, Idola Saint-Jean, l’insoumise (Montréal, Boréal, 2017), 382 p.
}

\section{Louise Bienvenue}

Volume 72, numéro 4, printemps 2019

URI : https://id.erudit.org/iderudit/1065052ar

DOI : https://doi.org/10.7202/1065052ar

Aller au sommaire du numéro

Éditeur(s)

Institut d'histoire de l'Amérique française

ISSN

0035-2357 (imprimé)

1492-1383 (numérique)

Découvrir la revue

Citer ce compte rendu

Bienvenue, L. (2019). Compte rendu de [Lavigne, Marie et Michèle

Stanton-Jean, Idola Saint-Jean, l'insoumise (Montréal, Boréal, 2017), 382 p.]

Revue d'histoire de l'Amérique française, 72(4), 105-108.

https://doi.org/10.7202/1065052ar d'utilisation que vous pouvez consulter en ligne.

https://apropos.erudit.org/fr/usagers/politique-dutilisation/ 
toriographie consistante dans le domaine. Contrairement à ce qu'avance l'auteure, l'historiographie missionnaire n'est pas sous le boisseau depuis 1962. En particulier, l'ouvrage de Chantal Gauthier sur les MIC (Femmes sans frontières, 2008), loin d'être "hagiographique ou apostolique», est une contribution originale, novatrice et forte, qui comprend, entre autres, l'analyse d'un questionnaire complété par 362 sœurs sur des questions comme l'entrée en religion ou les effets de Vatican II sur ces religieuses. Cet ouvrage important aurait mérité ici un meilleur sort.

La présentation d'Au risque de la conversion est alerte et vivante, mais le rejet des notes en fin d'ouvrage, avec renvois à la bibliographie, est un inconvénient d'autant plus pénible que plusieurs de ces notes sont substantielles: en tout 73 pages de notes, soit le quart de l'ouvrage. Globalement, toutefois, Catherine Foisy a tenu son pari: elle fait entrer par la grande porte l'histoire du mouvement missionnaire au cœur de l'histoire du Québec autour de la Révolution tranquille. Les témoignages des missionnaires, pris individuellement, ont une valeur limitée, à cause de ce que l'auteure appelle "des interprétations rétrospectives», mais pris dans leur ensemble et si bien situés dans leur contexte, ils nous livrent un portrait vivant et saisissant d'une facette incontournable de l'histoire du Québec.

GuY LAPERRIÈRE

Département d'histoire Université de Sherbrooke

Lavigne, Marie et Michèle Stanton-Jean, Idola Saint-Jean, l'insoumise (Montréal, Boréal, 2017), $382 \mathrm{p}$.

"La logique démocratique veut que les derniers vestiges de l'aristocratie disparaissent, je veux dire l'aristocratie des sexes» (p. 160). Ainsi s'exprimait Idola Saint-Jean, en 1928, dans un discours devenu célèbre. Sa vie durant, cette féministe montréalaise n'aura de cesse de défendre la cause de l'égalité entre hommes et femmes, s'exprimant dans un style plus radical que plusieurs de ses contemporaines. Dans cette passionnante biographie écrite à quatre mains, Marie Lavigne et Michèle Stanton-Jean racontent la trajectoire de cette singulière militante. Ne dissimulant pas leur admiration envers celle qu'elles appellent tout simplement Idola, les 
auteures cherchent à "réparer une grave injustice» en faisant la lumière sur une figure politique tombée «dans l'oubli» (p. 14).

La mémoire d'Idola Saint-Jean (1879-1945) n'est peut-être pas aussi négligée que l'affirment Lavigne et Stanton-Jean. Après tout, la féministe figure désormais dans les manuels scolaires et sa statue est bien en vue devant le Parlement de Québec. Mais ce que l'on connaît d'elle se limite souvent au combat suffragiste qu'elle a mené pendant deux décennies. Dans cet ouvrage étoffé mais facile d'accès, d'autres facettes de SaintJean sont mises en lumière, brossant de la sorte un portrait plus complexe et fascinant du personnage. On rencontre ainsi l'artiste de théâtre, la professeure de diction, la candidate politique ainsi que la journaliste de la radio et de la presse écrite.

Suivant une logique mi-chronologique, mi-thématique, l'ouvrage se divise en pas moins de 34 chapitres, rassemblés en 4 grandes parties. La première, "Trouver sa place au soleil», aborde les années de formation de la jeune femme. Saint-Jean grandit dans une famille aisée, d'allégeance libérale, dont elle hérite des principes. Elle fait de bonnes études au couvent Villa Maria, puis à l'Académie Saint-Urbain. Comme d'autres gens de son rang, elle passe ses vacances estivales à Cacouna, où elle côtoie entre autres le poète Émile Nelligan. Si l'engagement de son père au sein du Parti libéral est une source évidente d'inspiration pour elle, le véritable rêve d'Idola est le théâtre. La jeune femme s'impose d'ailleurs comme «l'une des plus délicieuses étoiles» de la scène nationale (p. 71). Le décès subit de son père en avril 1900 change la donne. Les finances familiales fragilisées, l'artiste, qui n'envisage aucunement le mariage, doit trouver des revenus. L'Université McGill l'engagera pour enseigner la diction française.

La deuxième partie de la biographie s'intéresse à la «Femme moderne et autonome». On y dépeint l'activité de Saint-Jean comme pionnière du féminisme. Celle-ci fonde, entre autres, l'éphémère Association artistique des dames canadiennes, attachée à la Fédération nationale SaintJean-Baptiste en 1908. Ses talents de conférencière sont mis à profit dans différentes causes défendues par la Fédération, comme la lutte à la mortalité infantile. Idola s'occupe aussi du comité de secours français lors de l'épidémie de grippe espagnole de 1918 et s'engage pour l'amélioration du sort des jeunes délinquants. La question du vote des femmes retient, bien sûr, l'attention des auteures. En 1922, Idola Saint-Jean devient secrétaire du nouveau Comité provincial pour le suffrage féminin. Les suf- 
fragistes doivent alors faire face à une opposition musclée du gouvernement Taschereau et de l'épiscopat catholique. Dans cette tempête, la militante semble inébranlable. Elle propage entre autres ses idées sur les ondes de CKAC, à partir de 1925. À l'automne 1929, elle est nommée directrice de la page féminine du Montreal Herald où elle tient, pendant un an, une étonnante page féminine, rédigée dans les deux langues. Les questions relatives aux droits des femmes y supplantent les thèmes habituels, comme la cuisine et le tricot. En 1933, Saint-Jean fonde son propre journal, La Sphère féminine/Women's Sphere, résolument féministe. Si, en raison de sources disponibles, les biographes mettent surtout l'accent sur la vie publique d'Idola, quelques documents permettent d'évoquer des événements de sa vie intime: une peine d'amour, vécue à l'âge de 44 ans, ainsi qu'une demande en mariage refusée au nom de sa nécessaire liberté.

"Changer l'ordre établi» est le titre de la troisième partie qui retrace l'évolution de la campagne suffragiste et observe les tensions entre les différentes sensibilités féministes dans un contexte hostile à la cause. Les auteures racontent évidemment la fondation de l'Alliance canadienne pour le vote des femmes du Québec, qu'Idola met sur pied en 1927 afin, dit-elle, de dégager son combat suffragiste de toute allégeance politique. On évoque aussi les autres luttes de celle qui concevait le féminisme comme un mouvement inéluctable: amélioration du statut juridique de la femme mariée (qu'elle compare à celui d'esclave), défense du droit au travail des femmes, revendication d'une pension aux mères célibataires, etc. Un chapitre rappelle aussi que Saint-Jean fut la première Canadienne française à se porter candidate à des élections fédérales en 1930. Preuve de sa notoriété, son nom circule au moment de nommer une première femme au Sénat.

La quatrième partie, plus courte, est consacrée aux dernières années de vie de la féministe, après l'obtention du suffrage provincial en 1940. On ne connait pas la cause de son décès en 1945, mais on sait que son cercueil fut porté uniquement par des femmes.

Célébrant surtout l'insoumise «qui refuse de se plier aux stratégies tout en douceur des féministes au langage réservé comme Marie Gérin-Lajoie, ou au ton diplomatique, comme Thérèse Casgrain " (p. 13), l'ouvrage propose de bonnes mises en contexte et s'appuie assez solidement sur l'historiographie. L'analyse de la pensée d'Idola sur les questions du féminisme, de la démocratie et de la citoyenneté y est toutefois menée trop furtive- 
ment; elle aurait pu faire l'objet de développements plus longs en tirant davantage profit, par exemple, des réflexions menées par Diane Lamoureux. Une discussion plus soutenue de l'efficacité stratégique des choix opérés par Saint-Jean aurait été aussi pertinente: son insoumission a-telle eu un prix politique? La lecture laisse parfois penser que la féministe, tout en parlant au nom de l'Alliance, faisait souvent cavalier seul. On sait peu de choses, en réalité, des autres membres de l'association fondée par Saint-Jean, au point où l'on se demande s'il ne s'agissait pas d'un cercle très étroit, dans les faits. Une sorte de flou plane sur cette organisation et son ampleur réelle, qu'il semble impossible de dissiper puisque ni les procèsverbaux ni les rapports financiers n’ont été conservés (p. 195). C’est donc souvent à partir de sources émanant de la présidente elle-même ou de son journal que les auteures évoquent les faits d'armes de l'Alliance. Or SaintJean était une habile propagandiste, toujours prête à promouvoir ses propres initiatives en comptant sur l'effet synergique des différentes tribunes où elle s'exprimait. Pour cette raison, une critique plus poussée des sources aurait été opportune. Par ailleurs, si on ne doute pas que l'ouvrage soit bien documenté, le travail de référencement n'est pas toujours aussi systématique que souhaité. Ces quelques réserves ne devraient pas, cependant, porter ombrage à cette biographie avant tout instructive qui retrace une trajectoire de vie tout à fait remarquable.

Louise Bienvenue Département d'histoire Université de Sherbrooke

Roulet, Éric, La Compagnie des îles de l'Amérique 1635-1651. Une entreprise coloniale au XVII siècle (Rennes, Presses universitaires de Rennes, 2017), 812 p.

Alors qu'ils s'intéressent depuis longtemps aux débuts de l'histoire de la Nouvelle-France, les historiens ont prêté peu d'attention aux Antilles françaises au XVII ${ }^{\mathrm{e}}$ siècle (à l'exception notable de Philip Boucher, de Paul Butel, d’Éric Schnakenbourg et de Jacques Petitjean Roget). Les sources, dit-on, manquent. Cet ouvrage d'Éric Roulet nous montre en revanche la richesse de la documentation sur la Compagnie des îles de l'Amérique, l'entreprise qui supervisa la conquête et l'exploitation des îles au nom de la France de 1635 à 1651. En effet, il s'agit d'une étude exhaustive de la compagnie, sa structure, son fonctionnement, son per- 